\title{
Advancing Metal-Phenolic Networks for Visual Information Storage
}

Qiong Dai, ${ }^{\dagger}$ Qun Yu, ${ }^{\dagger}$ Yuan Tian,,$^{\dagger}$ Xiaolin Xie,,$^{\dagger}$ Aixin Song, ${ }^{\dagger}$ Frank Caruso, ${ }^{\dagger}{ }^{\S}$ Jingcheng Hao, ${ }^{*, \dagger}$ and Jiwei Cui ${ }^{*, \dagger}, \dot{\dagger}$

${ }^{\dagger}$ Key Laboratory of Colloid and Interface Chemistry of Ministry of Education, Shandong University, Jinan, Shandong 250100, China

\$State Key Laboratory of Microbial Technology, Shandong University, Qingdao, Shandong 266237, China

${ }^{\S}$ ARC Centre of Excellence in Convergent Bio-Nano Science and Technology, and the Department of Chemical Engineering, The University of Melbourne, Parkville, Victoria 3010, Australia

*Email: jhao@sdu.edu.cn (J. H.); jwcui@sdu.edu.cn (J. C.)

KEYWORDS: metal-phenolic networks, polyphenols, coordination, sustainable chemistry, iron gall ink 
ABSTRACT: We report a facile inking strategy for visual information storage (e.g., writing, printing and beyond), via surface modification of substrates with polyphenols and subsequent in-situ formation of metal-phenolic networks (MPNs) on the substrates. The reported technique has several advantages compared with current printing techniques. Diverse substrates can be used to fulfill the requirements for different applications (e.g., printing, writing, painting, stamping). A range of colors (e.g., yellow, blue, green) can be realized using different polyphenols (e.g., tannic acid, gallic acid, pyrogallol) and metal ions (e.g., $\mathrm{Cu}^{\mathrm{II}}, \mathrm{Fe}^{\mathrm{III}}, \mathrm{Ti}^{\mathrm{IV}}$ ). The disadvantages (e.g., ink precipitation, color fading) associated with writing or printing using traditional ink can be overcome. The obtained paintings can be easily removed by using acids enabling the recycling of substrates. The reported strategy provides a new avenue for the development of portable, non-toxic and green technologies for writing, printing and beyond, which expands the applications of MPN-based materials. 


\section{INTRODUCTION}

Writing and printing on papers have played a key role in visual information storage due to their convenience, stability, and simplicity. There are three basic elements determining the writing and printing outcomes: the ink, writing/printing techniques, and substrates (e.g., papers). Iron gall inks (IGIs), with plant tannins and iron(II) sulfate as main ingredients, have been extensively used since the Middle Ages in Europe. ${ }^{1,2}$ IGIs have been exploited for numerous important transcripts and drawings in the Western World $^{3}$ and are still currently used for the preparation of important document archives. Ferrous ion $\left(\mathrm{Fe}^{\mathrm{II}}\right)$ and tannins, as the two main components, can form a soluble and blue-black polyphenol-Fe ${ }^{\mathrm{II}}$ complex within the ink mixture. The dark and indelible black of the ink is then achieved, upon writing on the paper, via the oxidation of $\mathrm{Fe}^{\mathrm{II}}$ to ferric ion $\left(\mathrm{Fe}^{\mathrm{III}}\right)$ and subsequent reassembly of the polyphenol-Fe $\mathrm{Fe}^{\mathrm{III}}$ complexes in the mixture. ${ }^{3}$ As the polyphenol- $\mathrm{Fe}^{\mathrm{III}}$ complexes can readily precipitate from aqueous solutions, the introduction of strong acids and additives is necessary to avoid flocculation and achieve a stable ink. ${ }^{1,3-5}$ Nevertheless, the long-term use of IGIs can cause pens to jam and the low $\mathrm{pH}$ of IGIs can result in corrosion of the writing tools. In addition, IGIs have been shown to accelerate the degradation of cellulose, thereby affecting the quality of the

printed paper. ${ }^{1,4,5}$ Consequently, complicated and costly processes have been devised for the long-term preservation of documents written using IGIs. ${ }^{4-7}$ Traditional Chinese paintings (TCPs), using brush pens, rice papers/silk scrolls, and plant-based inks, has also been facing similar problems in terms of long-term storage. ${ }^{8}$ In addition to the degradation of the painted papers, color fading or color alteration is problematic in TCPs.

As the development of modern printing techniques, a range of printing processes, including inkjet printing, screen printing, gravure printing, flexographic printing and photo-printing, have been developed to fulfill the requirement of applying functional inks with diverse properties. ${ }^{9,10}$ Therefore, a series of functional 2D materials, besides paper-based prints for information 
storage, can be printed, including conductive matrix, electronic or photonic devices, biosensors and detecting arrays. ${ }^{9-11}$ Although various soft matrix and substrates have been developed for printing processes, paper-based substrates still play an irreplaceable role in the improvement and exploitation of printing techniques. ${ }^{12}$

Over the years, traditional paper-based writing and printing strategies have also led to various economic and environmental issues that have necessitated the development of alternative convenient and environment-friendly techniques for daily writing, printing, and painting applications. ${ }^{1,13-15}$ For temporary recording purposes, several types of rewritable papers have been developed. For instance, the rewritable photonic paper was developed through magnetic field-induced self-assembly and fixation of $\mathrm{Fe}_{3} \mathrm{O}_{4} @ \mathrm{SiO}_{2}$ colloids within a poly(ethylene glycol) diacrylate matrix. ${ }^{16}$ Such a photonic paper system has potentials for outdoor applications such as posters or signage, but is not suitable for daily writing or printing of documents since ordinary writing paper is not a compatible substrate for fixation of the photonic paper. Alternatively, a rewritable paper based on the isomerization of spiropyrans in aqueous solution could serve as an environment-friendly printing substrate. ${ }^{14}$ Such rewritable papers developed with the ordinary printing paper as the basic substrate were suitable for daily water-jet printing using a commercial desktop printer. The advantage of this method is that the prints could be erased and rewritten easily. However, the prints had a lifetime of $22 \mathrm{~h}$, thus limiting their usage in applications requiring long-term storage. The retaining time of the prints could be prolonged to over one month by incorporating polyvinyl alcohol into the rewritable paper. ${ }^{17}$ However, the issue of time-dependent color fading were still existing. This technique was then improved by Huang and co-workers who constructed a water-jet rewritable paper based on metal-ligand coordination to extend the print lifetime to over six months. ${ }^{15}$ However, the four-layer paper required several repeated painting and drying cycles, making it time and labor intensive. In addition, the recycling process of the rewritable paper required the usage of fluoride containing 
toxic chemicals (e.g., tetrabutylammonium fluoride). Rewritable papers based on photochromic compounds usually required complicated synthesis process, special photo-printing tools, or decoloring processes with high energy consumption, limiting practical applications. ${ }^{18-20}$

Metal-phenolic networks (MPNs) are an emerging class of materials that are based on metalligand coordination. ${ }^{2,21}$ These materials have been exploited for the assembly of a vast library of functional materials. ${ }^{2,22-25}$ For instance, a range of self-healing hydrogels, ${ }^{24,26,27}$ protective surface coatings, ${ }^{28-30}$ and nanostructured cargo carriers ${ }^{2,31}$ have been designed based on MPN systems. Moreover, a series of colors, including grey, brown, yellow, orange, purple, and green, can be generated upon the formation of MPNs using different metal ions. ${ }^{26,31}$ The intrinsic color and high biocompatibility of MPNs, and their adhesion to a wide range of substrates make them potential candidates for diverse coloration applications. In addition, the $\mathrm{pH}$-responsiveness of MPNs can be exploited to achieve deinking of the substrates. Herein, we develop a strategy taking advantage of the intrinsic color, versatility, and reversibility of MPN systems for a wide range of inking applications (e.g., writing, printing, TCP, stamping, and slogan spraying), as an alternative to traditional strategies. In general, owing to the adhesiveness of polyphenols to a wide range of substrates, ${ }^{32-34}$ polyphenol coatings can be easily formed on a substrate via simply spraying polyphenol solutions onto the substrate. The polyphenol-modified substrate (Psubstrate) with a colorless polyphenol coating can then be used for painting by using solutions containing metal ions as inks (M-inks) (Scheme 1) via a series of application methods. In addition, deinking can be easily achieved by subjecting the substrates to acidic solutions, thereby allowing recycling of substrates (Scheme 1). The present technique can overcome the disadvantages associated with the use of IGIs, including pens jamming/blocking, precipitation of inks after long-term storage, and corrosion of the writing tools after long-term usage. 


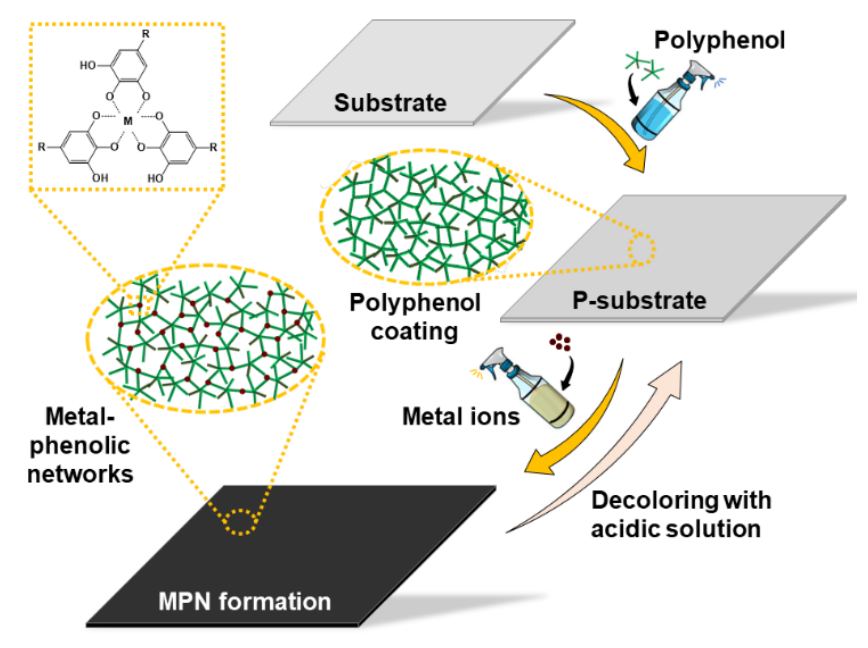

Scheme 1. Schematic illustration of inking on substrates via the formation of MPNs. A Psubstrate is obtained by spraying polyphenol solutions onto the substrates. A thin layer of polyphenol coating is formed after solvent evaporation. Metal ion solutions used as M-inks are applied onto the P-substrate, and ink prints develop onto the substrate upon formation of MPNs. Deinking of the substrate can be achieved by washing the substrate with acidic solutions.

\section{EXPERIMENTAL SECTION}

2.1 Materials. Tannic acid (TA, ACS reagent) and titanium(IV) bis(ammonium lactato)dihydroxide solution (Ti-BALDH, 50 wt.\% in $\mathrm{H}_{2} \mathrm{O}$ ) were purchased from SigmaAldrich (China). Gallic acid (GA, 99\%, from Rhus chinensis Mill), ethyl gallate (EG, 98\%), pyrogallol (PG, 99\%), (+)-catechin hydrate (CC, 98\%), 2-thenoyltrifluoroacetone (TTA, 99\%), nickel(II) chloride hexahydrate $\left(\mathrm{NiCl}_{2} \cdot 6 \mathrm{H}_{2} \mathrm{O}\right)$, europium(III) chloride hexahydrate $\left(\mathrm{EuCl}_{3} \cdot 6 \mathrm{H}_{2} \mathrm{O}\right)$, manganese(II) chloride tetrahydrate $\left(\mathrm{MnCl}_{2} \cdot 4 \mathrm{H}_{2} \mathrm{O}\right)$, terbium(III) chloride hexahydrate $\left(\mathrm{TbCl}_{3} \cdot 6 \mathrm{H}_{2} \mathrm{O}\right)$, and cerium(III) chloride $\left(\mathrm{CeCl}_{3}\right.$, anhydrous) were purchased from J\&K Scientific (China). Acetylacetone (AA, 99\%), iron(III) chloride hexahydrate $\left(\mathrm{FeCl}_{3} \cdot 6 \mathrm{H}_{2} \mathrm{O}\right)$, calcium(II) chloride $\left(\mathrm{CaCl}_{2}\right.$, anhydrous), and cobalt(II) chloride hexahydrate $\left(\mathrm{CoCl}_{2} \cdot 6 \mathrm{H}_{2} \mathrm{O}\right)$ were purchased from Aladdin (China). Copper(II) chloride dihydrate $\left(\mathrm{CuCl}_{2} \cdot 2 \mathrm{H}_{2} \mathrm{O}\right)$ and magnesium(II) sulfate $\left(\mathrm{MgSO}_{4}\right.$, anhydrous) were purchased from Sinopharm Chemical 
Reagent (China). All materials were used as received without further purification, unless otherwise stated.

2.2 Preparation of Coordinated Rare Earth Metal Complexes. The Eu-TTA complex was synthesized according to published methods. ${ }^{35}$ First, $266 \mathrm{mg}$ TTA was dissolved in $6 \mathrm{~mL}$ ethanol, followed by the addition of $1.2 \mathrm{~mL}$ of $1 \mathrm{M} \mathrm{NaOH}$. Then, $146 \mathrm{mg} \mathrm{EuCl}{ }_{3} \cdot 6 \mathrm{H}_{2} \mathrm{O}$ was dissolved in $42 \mathrm{~mL}$ water and mixed with the above solution. The mixture was heated at $60{ }^{\circ} \mathrm{C}$ for $1 \mathrm{~h}$ before cooling to room temperature, generating a yellow precipitate during the cooling process. The precipitate was recovered by filtration and washing with water. A yellow powder, Eu-TTA complex, was obtained via drying the precipitate under vacuum.

The $\mathrm{Tb}-\mathrm{AA}$ complex was synthesized in a $1.5 \mathrm{~mL}$ tube according to a published method. ${ }^{31}$ $\mathrm{TbCl}_{3} \cdot 6 \mathrm{H}_{2} \mathrm{O}$ and AA were dissolved separately in ethanol to a final concentration of $240 \mathrm{mM}$. Then, $200 \mu \mathrm{L}$ Tb solution was mixed with $400 \mu \mathrm{L}$ AA solution, followed by the addition of 30 $\mu \mathrm{L}$ of $1 \mathrm{M} \mathrm{NaOH}$. The mixture was vortexed thoroughly and then heated at $45{ }^{\circ} \mathrm{C}$ under sonication for $4 \mathrm{~h}$ until the solution became transparent. The solution was allowed to cool to room temperature and used as prepared. The emission spectra of Eu-TTA and Tb-AA at 312 nm were shown in Figure S1.

2.3 Preparation of Polyphenol-Modified Substrates (P-substrates). Various polyphenol solutions (100 $\left.\mathrm{mg} \mathrm{mL}^{-1}\right)$ were first prepared by dissolving different polyphenols in ethanol. For polyphenols with good water solubility (e.g., TA, EG, PG, and SA), water could be used to replace ethanol for the preparation of $100 \mathrm{mg} \mathrm{mL}^{-1}$ polyphenol solutions without influences on the results. The polyphenol solutions were then sprayed onto different substrates using a spray bottle. The substrates were allowed to air-dry at room temperature to obtain P-substrates with polyphenol coatings. 
2.4 Printing. To prepare the Fe-ink $\left(100 \mathrm{mg} \mathrm{mL}^{-1}\right)$ for the printing experiment, $2 \mathrm{~g} \mathrm{FeCl}_{3} \cdot 6 \mathrm{H}_{2} \mathrm{O}$ was dissolved in $20 \mathrm{~mL}$ ethanol. The Fe-ink $(15 \mathrm{~mL})$ was poured in an emptied HP 803 ink cartridge that had been washed with water and ethanol repeatedly. The cartridge was then placed in a HP DeskJet 1112 printer for printing on P-papers.

2.5 Characterization. The microscopic structures of the paper surfaces with or without polyphenol or MPN coatings were observed via scanning electron microscopy (SEM) on a Zeiss G300 microscope. Prior to SEM characterization, the papers were cut into small pieces $(5 \mathrm{~mm}$ $\times 3 \mathrm{~mm}$ ), mounted on silicon wafers, and then sputter-coated with gold. Papers with or without polyphenol or MPN coatings were cut into pieces $(4 \mathrm{~cm} \times 4 \mathrm{~cm})$. The reflection visible spectra and UV-vis absorption spectra of the papers were obtained on a UV-vis spectrophotometer (Shimadzu, UV-2600).

2.6 Decoloration. The papers with MPN coatings were immersed in $\mathrm{HCl}$ solution at $\mathrm{pH} 2$ for $5 \mathrm{~min}$, then transferred into water for 5 min washing to remove the MPN residues and excess acids. The papers were then immersed in $\mathrm{NaOH}$ solution at $\mathrm{pH} 9$ for 5 min and transferred into water for another $5 \mathrm{~min}$ washing to remove the excess base solutions. The papers were allowed to air-dry at room temperature before re-utilization.

\section{RESULTS AND DISCUSSION}

The influence of polyphenol concentration on the polyphenol-modified papers (P-papers) was investigated via spraying tannic acid (TA) at different concentrations (10, 50, 100, 150, and 200 $\mathrm{mg} \mathrm{mL} \mathrm{m}^{-1}$ ) on the ordinary printing paper. A concentration of $100 \mathrm{mg} \mathrm{mL}^{-1}$ was determined as the most appropriate polyphenol concentration - a relatively deep black was obtained upon Mink application and the external features of the paper were unchanged (Figures S2 and S3). Iron chloride $\left(\mathrm{FeCl}_{3}\right)$ solution was selected as the ink component for the formation of black ink $(\mathrm{Fe}-$ ink). TA-Fe ${ }^{\mathrm{III}}$ networks result in dark black (Figures 1 and S3). The concentration of Fe-ink 
was varied as the concentration of TA remained constant, and reflection visible spectrometry was used to determine the lowest concentration of Fe-ink $\left(100 \mathrm{mg} \mathrm{mL}^{-1}\right)$ required to achieve the deepest black (Figure S3). The black paintings developed on P-paper using Fe-ink was comparable to the black obtained using commercial ink (C-ink) (Figure 1A). Although there were negligible changes for the macroscopic features of P-papers compared with the untreated paper, microscopic feature changes of papers with or without TA treatment were observed from scanning electron microscopy (SEM) images (Figure 1B). The loosely interlaced cellulose fibers with heterogeneous porosity were covered by a layer of TA coating, resulting in a more homogeneous surface with no obvious pores (Figure 1B). Different treatments also led to the variation in hydrophobicity of the substrates. In particular, the application of Fe-ink on the untreated paper resulted in a more hydrophobic surface, while the application of TA on the untreated paper resulted in a more hydrophilic surface, which was even more hydrophilic upon the application of Fe-ink (Figure 1B).
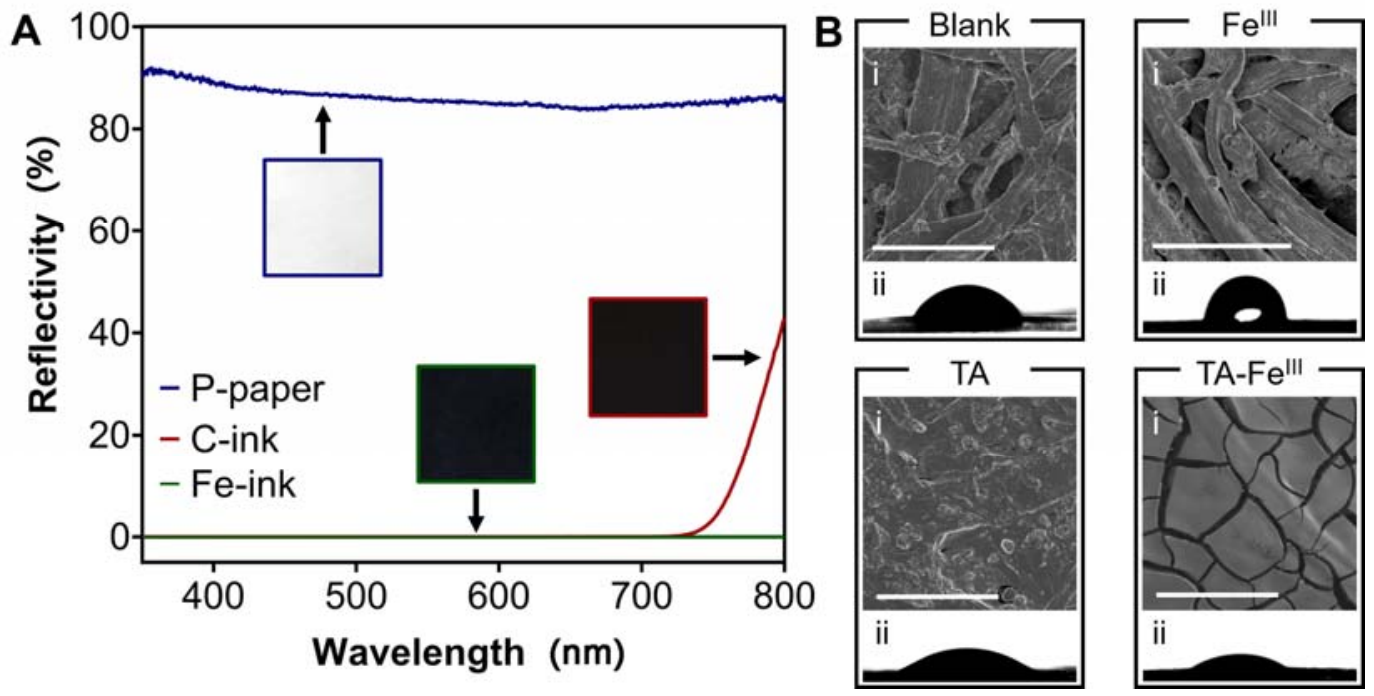

Figure 1. (A) Reflection visible spectra of the paper treated with TA solution (P-paper, blue line), and the P-papers that were subsequently treated with $\mathrm{FeCl}_{3}$ solution ( $\mathrm{Fe}$-ink, green line) or commercial ink (C-ink, red line). (B) (i) SEM images of untreated paper (blank) and papers treated with solutions of $\mathrm{Fe}^{\mathrm{III}}$, TA, as well as both $\mathrm{TA}$ and $\mathrm{Fe}^{\mathrm{III}}$. Scale bars are $50 \mu \mathrm{m}$. (ii) Images 
of water droplets on papers subject to different treatments. Contact angle values of papers are $54^{\circ}$ (Blank), $80^{\circ}\left(\mathrm{Fe}^{\mathrm{III}}\right), 41^{\circ}(\mathrm{TA})$, and $31^{\circ}\left(\mathrm{TA}-\mathrm{Fe}^{\mathrm{III}}\right)$, respectively. A summarizing table with all contact angle values of papers with various treatments is shown in Table S1.

In addition, the ink paints developed on P-paper using Fe-ink could be erased easily and conveniently using acidic solutions (hydrochloric acid at $\mathrm{pH} 2$ ), which was induced by the disassembly of MPN complexes, thus enabling reuse of the P-paper (Figure 2A). This easy and convenient decoloring method could reduce the treatment cost of wasted prints. The in-situ formation and disassembly of MPN complexes on papers were monitored by ultra-violetvisible (UV-vis) spectroscopy (Figure 2B). The ligand-to-metal charge transfer (LMCT) band characteristics of P-papers with Fe-ink prints (Figure 2B, blue line) indicated the formation of TA-Fe ${ }^{\mathrm{III}}$ coordination complexes ( $\sim 565 \mathrm{~nm}$, the dominant is bis-type complexation) $)^{24,36,37}$ while, the disappearance of the LMCT band for papers with Fe-ink prints erased by acids (Figure 2B, green line) confirmed the disassembly of MPN complexes. Apart from coordinating to the polyphenol layer on P-papers, the $\mathrm{Fe}^{\mathrm{III}}$ could also interact with the cellulose in the substrate (Figure 2B, yellow line) which further increased the stability of MPN-based prints on the substrate. 

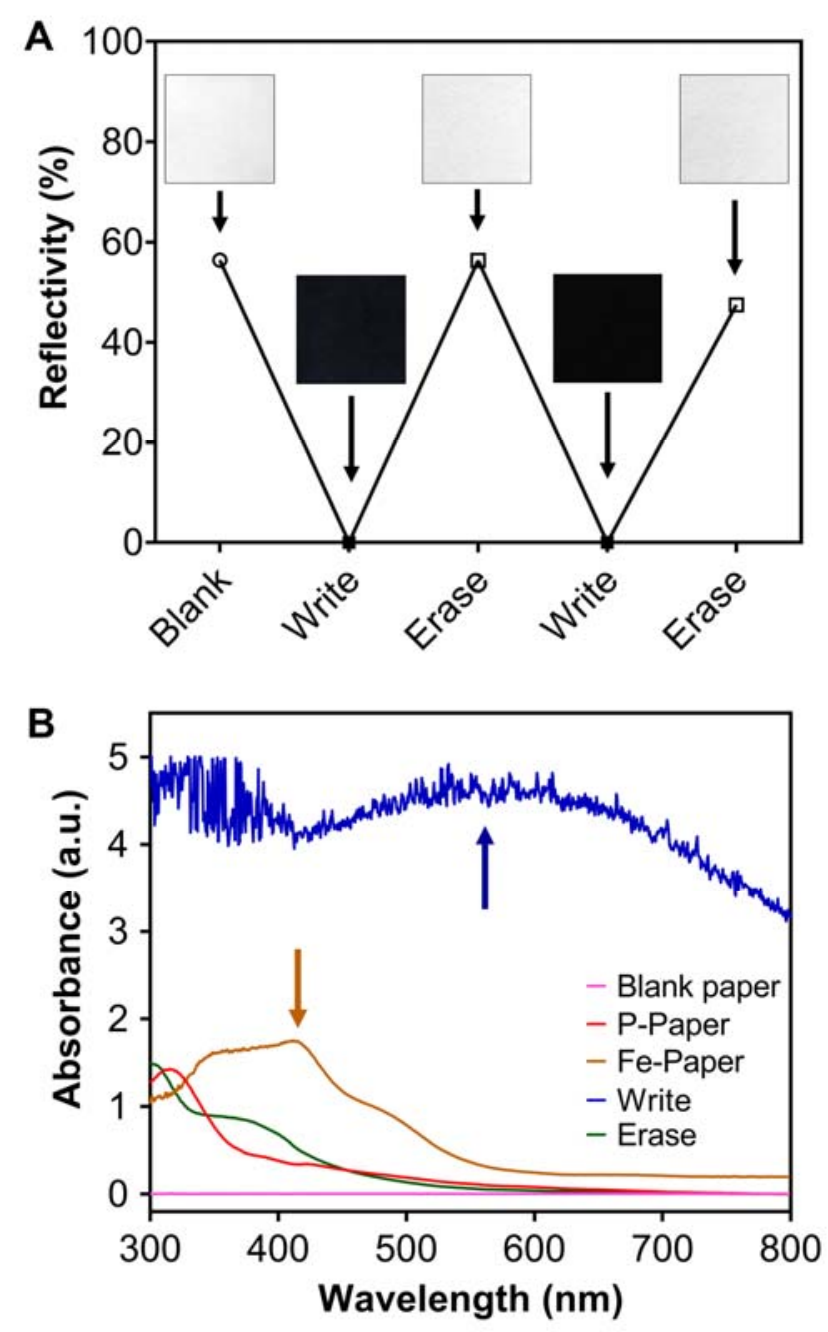

Figure 2. (A) Plot of the reflectivity at $555 \mathrm{~nm}$ as a function of writing/erasing cycles of the Ppaper through Fe-ink spraying (writing) and $\mathrm{HCl}$ immersion (erasing). Insets are photographs of the papers $(40 \mathrm{~mm} \times 40 \mathrm{~mm})$ examined. (B) UV-vis absorption spectra of papers with or without diverse treatment processes. The yellow and blue arrows indicate the Fe ${ }^{\mathrm{III}}$ LMCT band of papers with Fe-ink only (yellow) and with TA-Fe ${ }^{\mathrm{III}}$ prints (blue), respectively.

Plant polyphenols, with TA as a typical example, belong to a large family of natural compounds. In the present study, six types of polyphenols, TA, gallic acid (GA), ethyl gallate (EG), pyrogallol (PG), catechin (CC), and methylsalicylic acid (SA), were examined to assess their potential in preparing P-papers for practical applications. All polyphenols were soluble in ethanol, resulting in transparent solutions ranging from colorless to light yellow, and formed 
MPN coatings on papers upon interaction with Fe-ink applied via spraying (Figures 1B, 3A, and S4). Changes in surface microstructures of the P-papers without or with Fe-ink were imaged by SEM (Figure S5). There was no obvious difference between surface morphologies of blank papers before and after spraying of diverse polyphenol solutions. However, microscopic structures as well as hydrophobicity of paper surfaces with or without different polyphenol coatings were different (Figures 1B, S3, and S6), indicating that the hydrophobicity of substrates with various treatment was dependent on the physicochemical properties, including surface chemistry and the microscopic structure, of the substrate surfaces. While irrespective of the polyphenol (primary) coating applied, the coating on the surface of the P-paper became denser and thicker after spraying of Fe-ink, indicating the formation of MPNs (Figures 1B, S4).

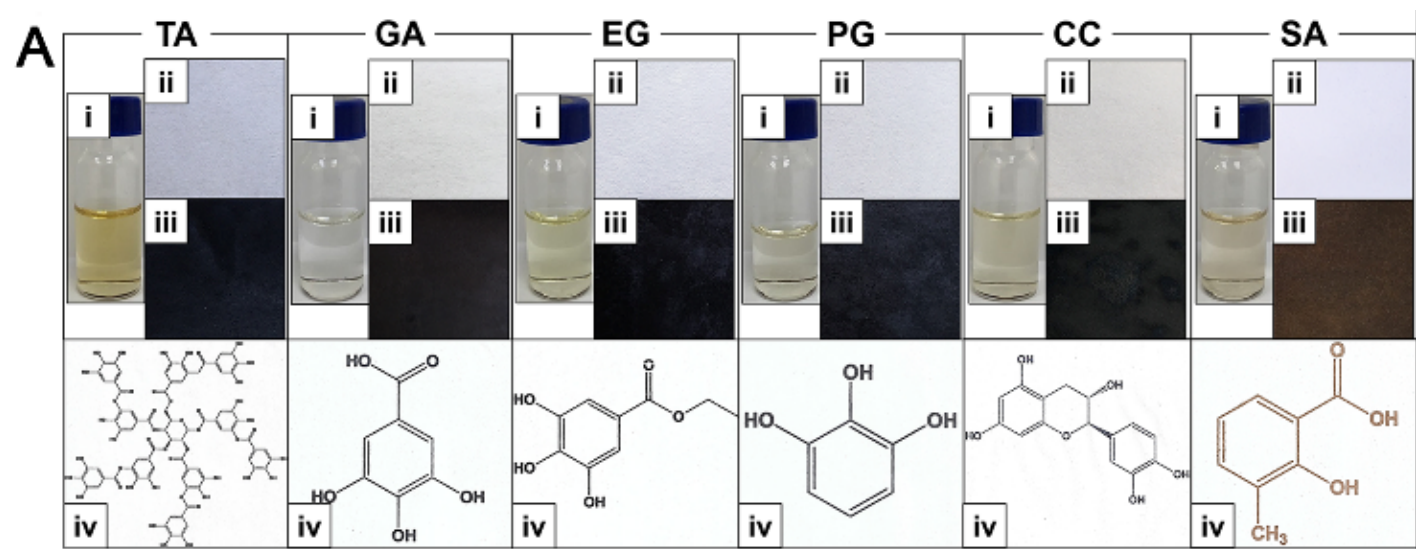

B
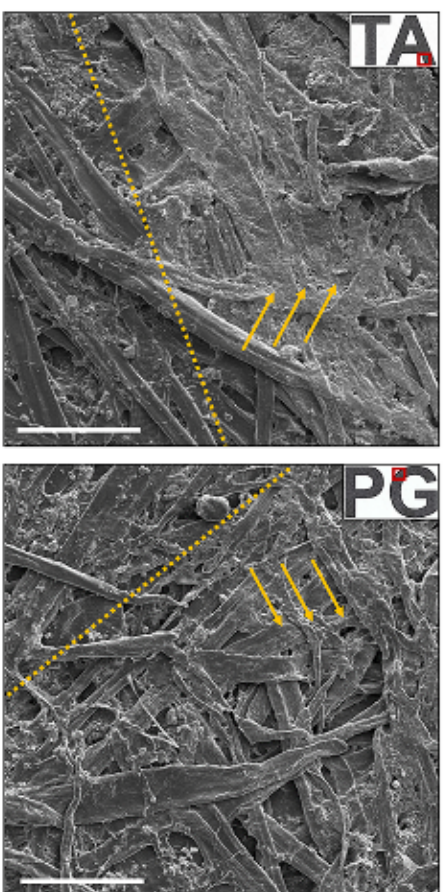
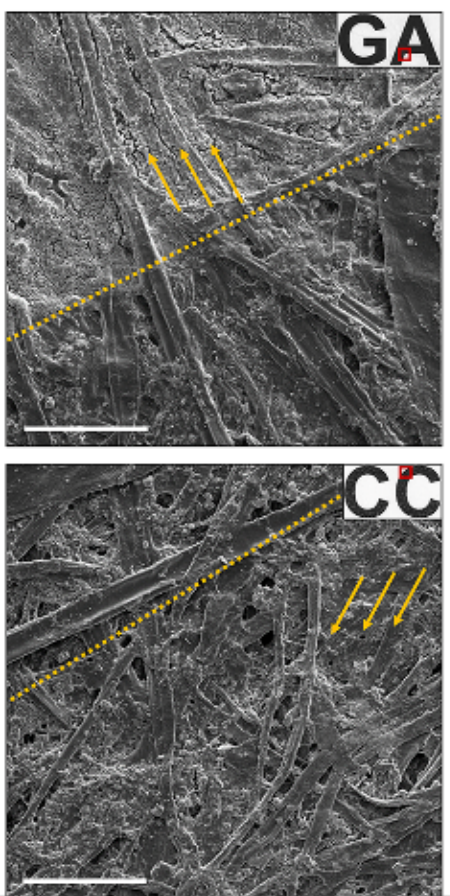
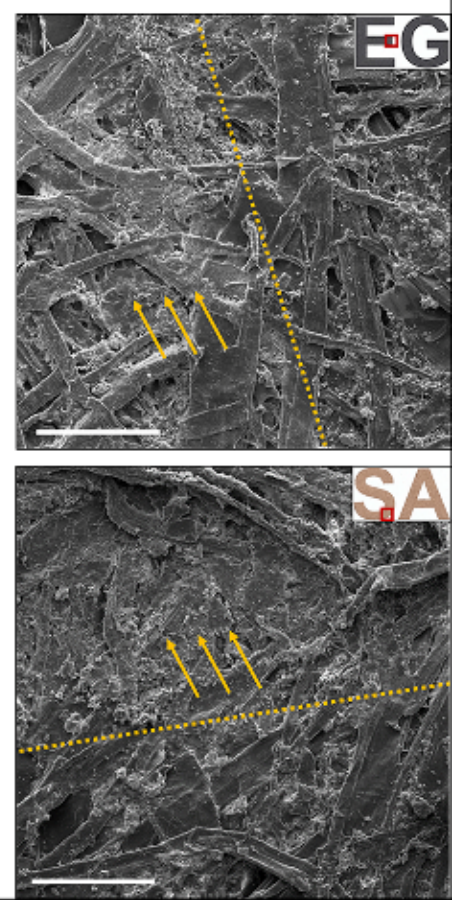
Figure 3. (A) Polyphenol solutions (i) were sprayed on ordinary printing papers to generate Ppapers (ii), which were then sprayed with Fe-ink for visual paintings (iii). Molecular structures (iv) of various polyphenols were printed with a HP DeskJet 1112 printer with cartridges (HP 803) refilled with Fe-ink on P-papers treated with different polyphenol solutions. (B) SEM images of prints obtained using a HP DeskJet 1112 printer with cartridges refilled with Fe-ink. The prints were generated on P-papers treated with different polyphenols as abbreviated in the insets. The yellow lines separate areas with and without Fe-ink coverage; the arrows indicate the area with Fe-ink coverage. Scale bars are $100 \mu \mathrm{m}$. The red squares in the insets indicate the regions that were analyzed for SEM analysis.

Regardless of the M-ink application method (spraying or printing), distinct differences could be observed between surfaces of P-papers with and without Fe-ink prints (Figures 3B and S5). However, compared to the spraying method which introduced a large amount of $\mathrm{Fe}^{\mathrm{III}}$ onto the P-papers allowing the formation of a thick MPN coating on the papers (Figures 1B and S4), the printing method could only introduce a small amount of $\mathrm{Fe}^{\mathrm{III}}$ onto the P-papers which was enough for color development but not enough for the formation of thick MPN coatings covering the original cellulose fiber structures of the papers (Figures 3B and S5). Furthermore, SEM analysis revealed a much thinner layer of SA-Fe coating compared with the other polyphenolFe coatings (Figure S4). Macroscopically, the in situ formation of SA-Fe complexes resulted in a dark brown color, whereas that of complexes comprising other polyphenols resulted in dark black (Figure 3A). These results indicate that the catechol or galloyl groups are required for generating the stable black paintings on substrates. As numerous plant polyphenols ${ }^{32,33}$ can fulfill this requirement, there exists a large library of potential candidates that can be used as substrate pre-treatment agents for this technique.

Fe-ink could also be applied onto P-papers via ink-jet printing (Figures 3A and 4) using, for instance, a commercial HP DeskJet 1112 printer (Figure S7). The quality of the prints using Fe- 
ink was comparable to that of the prints obtained using $\mathrm{C}$-ink prints (Figure 4A and 4B). In addition to generating high-quality prints, Fe-ink can be filled into a fountain pen for writing and drawing on P-papers without the risk of the pen jamming (Figure S8). Moreover, Fe-ink can be utilized as an alternative of the pad ink for stamping onto P-papers (Figure S9).

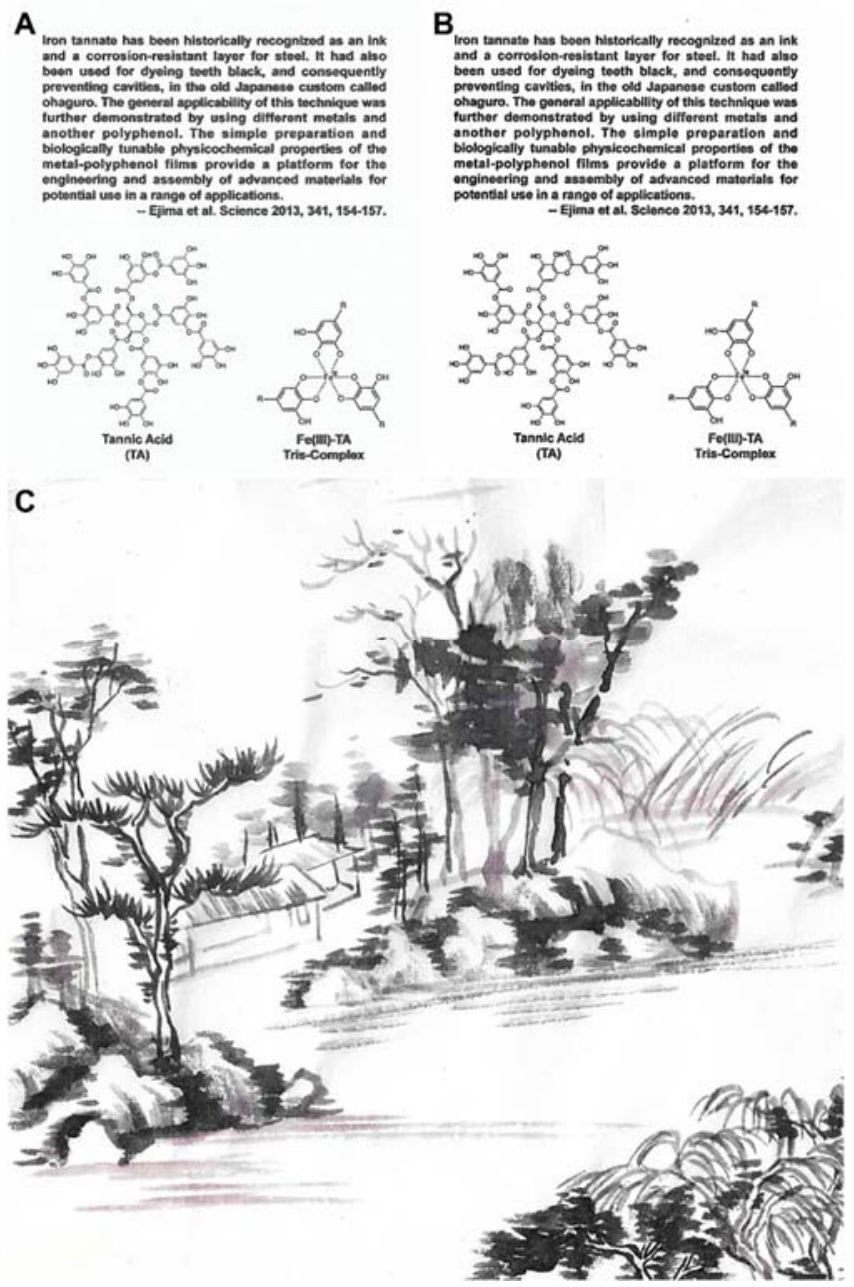

Figure 4. (A, B) Ink-jet prints $(210 \mathrm{~mm} \times 297 \mathrm{~mm})$ obtained on a HP DeskJet 1112 printer with cartridges (HP 803) filled with C-ink on untreated paper (A) or refilled with Fe-ink on TAtreated paper (B). (C) Traditional Chinese ink painting using a Chinese brush and Fe-ink on TA-treated rice paper.

Using traditional brush pens and Fe-inks at varying concentrations, different degrees of black, including typical dark black generated in $\mathrm{TCPs},{ }^{8}$ could be obtained (Figure $\mathrm{S} 10$ ). In addition to different hues of black, the present strategy produced a series of reddish-brown colors (Figure 
S10), thus enabling further versatility, diversity, and creativity in TCPs. The treatment of rice paper using polyphenol solutions generated a layer of polyphenol coating on their surfaces. This type of paper is suitable for creating traditional ink paintings using brush pens (Figures 4C).

Owing to the intrinsic adhesiveness of polyphenols to a wide range of substrates, ${ }^{32,33}$ the present painting technique could be applied to a series of diverse substrates, including paper, a polyvinyl chloride surface, a latex painted wall, leather, cotton or nylon cloth, and aluminum foil using different polyphenols (Figures 5A and S11). The MPN-based painting technology could further be extended to outdoor advertisement applications. For instance, advertising slogans or logos could be sprayed onto polyphenol-treated walls using M-inks, as demonstrated in Figure S12. The ink imprints generated on diverse P-substrates via different applying methods (including writing, ink-jet printing, painting, stamping and spraying) were stable for at least one year, with no obvious color changes. Notably, no additional treatment was required to achieve such stability.

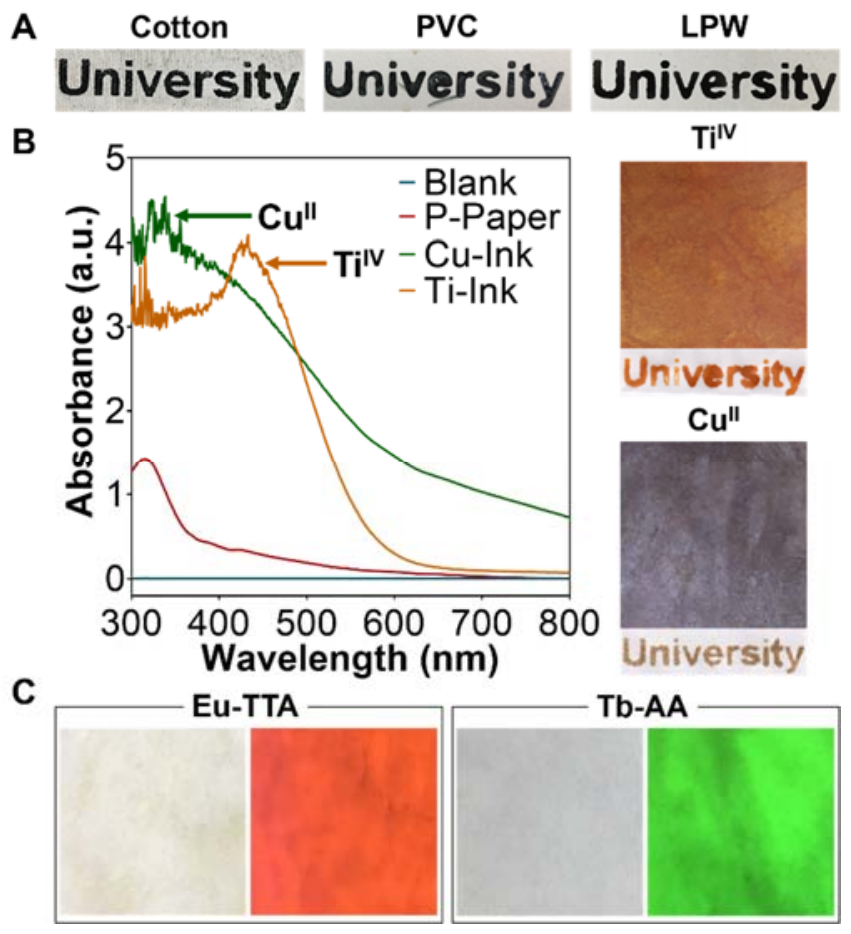

Figure 5. (A) Stampings on TA-treated cotton T-shirt, a polyvinyl chloride (PVC) surface, and a latex painted wall (LPW) using Fe-ink. (B) UV-vis absorption spectra (left) of papers with 
different treatments. The green and yellow arrows indicate the $\mathrm{Cu}^{\mathrm{II}}$ and $\mathrm{Ti}^{\mathrm{IV}} \mathrm{LMCT}$ band of $\mathrm{P}-$ papers with $\mathrm{Cu}$-ink and Ti-ink prints, respectively. Photographs (right) of TA-treated papers sprayed or stamped with Ti-ink or Cu-ink. (C) Photographs of TA-treated filter papers painted with $\mathrm{Eu}-\mathrm{TTA}$ and $\mathrm{Tb}-\mathrm{AA}$ solutions as inks under ambient light (left) and UV light (right), respectively.

As can be seen from Figures 5B and S13, a range of metal ions can be applied as inks for the generation of diverse colors. UV-vis absorption spectra (Figure 5B) showing typical LMCT band characteristics of $\mathrm{Ti}^{\mathrm{IV}}$ (Figure 5B, yellow line) and $\mathrm{Cu}^{\mathrm{II}}$ (Figure 5B, green line) indicated the formation of $\mathrm{TA}-\mathrm{Ti}^{\mathrm{IV}}$ and $\mathrm{TA}-\mathrm{Cu} \mathrm{u}^{\mathrm{II}}$ coordination complexes on papers with Ti-Ink or $\mathrm{Cu}-$ Ink prints. ${ }^{38-40}$ This confirmed that the development of diverse colors on P-papers was based on the in-situ formation of MPN complexes on the papers. These metal ion solutions, similar to Fe-ink, resulted in stable and colorful ink prints on P-papers, thus broadening the presented MPN-based painting technique to achieve multicolor paintings. Furthermore, different metal ions could be mixed to provide additional choices of colors (Figure S14). A screening test that involved stamping of different M-inks on P-papers revealed that a series of colors, including orange, brown, yellow, and pink, could be obtained (Figure S15). The mono- and mixed metal ion solutions examined are prototype M-inks that are useful for colorful printing applications for daily use. Moreover, using solutions of coordinated rare earth metal complexes (i.e., europium-2-thenoyltrifluoroacetone $(\mathrm{Eu}-\mathrm{TTA})$ and terbium-acetylacetone $(\mathrm{Tb}-\mathrm{AA})$ complexes) as inks, the visibility of the prints could be tailored depending on the light. For instance, paintings onto P-papers produced by brushing or stamping were invisible under ambient light but were fluorescent under UV light (Figures 5C and S16). Such a feature could be useful for anti-counterfeit applications. The present painting strategy could be also extended to an even broader range of practical applications, including room decoration and self-painted clothes. 
Unlike the oily and sticky C-inks that contain different organic and inorganic additives, ${ }^{41-43}$ the M-inks developed herein dissolve in aqueous solutions and are non-toxic, odorless, non-sticky, and can be easily washed off upon skin contact, thereby representing more user-friendly inks (Table S2). The direct incorporation of polyphenols into paper pulp during papermaking process is currently under investigation. In addition, the introduction of polyphenols into Ppapers and other P-substrates would provide the substrates with anti-bacterial and antioxidation properties, taking advantage of the intrinsic properties of the polyphenol coating. ${ }^{32}$ Additionally, compared with the pre-mixed IGI, the dosage of Fe-inks, using the present strategy, can be more easily controlled with fewer free iron ions remaining on the paper. The presence of free iron ions using IGI has been reported to be a major cause of the damage of cellulose in papers. ${ }^{1}$ Therefore, in the present approach, the degradation rate of papers caused by free iron ions can be largely reduced. This indicates that MPN-based printing and painting techniques are suitable for paper-based documentation or artworks that require long-term storage.

\section{CONCLUSIONS}

In summary, we have introduced a green and facile strategy for diverse practical applications including writing, printing, painting, and stamping that can overcome the disadvantages of IGIs and TCP inks. The present technique involves the polyphenol coating of substrates (to obtain P-substrates) and subsequent visual paintings via formation of MPNs upon contact of metal ion solutions as inks with the P-substrates. A series of natural plant polyphenols were shown to produce surface coatings on papers, generating P-papers without obvious changes in their macroscopic properties. Fe-inks were introduced onto P-papers using different methods, including printing, writing, drawing, painting, stamping, and spraying via the formation of MPNs. The visual paintings developed via MPN assembly is stable (more than 16 months as the time tested) without any further treatment and can be removed easily through the use of 
acidic solutions. Moreover, the black prints developed via this strategy are anticipated as permanent prints that are durable (e.g., last for years), as this strategy shares the same mechanism with the IGI, which has been widely accepted as a permanent ink for archiving. The high quality of the MPN-based prints and TCPs also demonstrates their potential for applications in daily print and artistic designs. Successful printing was also achieved on other substrates such as cotton, nylon, latex painted wall, polyvinyl chloride cupboard, and leather. MPN assembly additionally affords a broader variety of M-inks owing to its versatility in terms of the metals ions involved, allowing the development of diverse colors, expanding the prints from black and white to multicolor, as well as luminescence properties. The achievement of multicolor as well as multifunction via in situ formation of MPN structures on various substrates further expanded the application of this technique from daily writing, painting, and printing to room decoration, clothing design and dyeing, as well as outdoor advertisement.

The present strategy is anticipated to be useful for long-term storage of archive files or artworks and the easy recycle of papers for temporary records. It also provides a new avenue for the development of portable, non-toxic and green technologies for visual information storage via writing, printing and beyond. Furthermore, taking advantages of the diversity and modifiability of MPNs, the MPN-based printing strategy will facilitate the development of printable 2D materials and further expand the applications of MPN-based materials.

\section{ASSOCIATED CONTENT}

\section{Supporting Information}

The Supporting Information is available free of charge on the ACS Publications website. 
Additional experimental methods on the preparation of coordinated rare earth metal complexes and printing on P-papers, as well as additional data on the characterization and application of MPN layers on substrates (PDF)

\section{AUTHOR INFORMATION}

\section{Corresponding Authors}

*Email: jhao@sdu.edu.cn (J. H.)

*Email: jwcui@sdu.edu.cn (J. C.)

\section{ORCID}

Qiong Dai: 0000-0003-1510-6054

Qun Yu: 0000-0003-3145-4833

Aixin Song: 0000-0001-9766-1742

Frank Caruso: 0000-0002-0197-497X

Jingcheng Hao: 0000-0002-9760-9677

Jiwei Cui: 0000-0003-1018-4336

\section{Notes}

The authors declare no competing financial interest.

\section{ACKNOWLEDGMENT}

This research was funded by the National Natural Science Foundation of China (J.C., 21603120 and 21872085; Q.D., 21802088), China Postdoctoral Science Foundation (Q.D., 
2018M632662), Natural Science Foundation of Shandong Province (Q.D., ZR2018BB036; J.H., ZR2018ZA0547).

\section{REFERENCES}

(1) Rouchon, V.; Duranton, M.; Burgaud, C.; Pellizzi, E.; Lavédrine, B.; Janssens, K.; de Nolf, W.; Nuyts, G.; Vanmeert, F.; Hellemans, K. Room-Temperature Study of Iron Gall Ink Impregnated Paper Degradation under Various Oxygen and Humidity Conditions: TimeDependent Monitoring by Viscosity and X-Ray Absorption Near-Edge Spectrometry Measurements. Anal. Chem. 2011, 83, 2589-2597.

(2) Ejima, H.; Richardson, J. J.; Liang, K.; Best, J. P.; van Koeverden, M. P.; Such, G. K.; Cui, J.; Caruso, F. One-Step Assembly of Coordination Complexes for Versatile Film and Particle Engineering. Science 2013, 341, 154-157.

(3) Ponce, A.; Brostoff, L. B.; Gibbons, S. K.; Zavalij, P.; Viragh, C.; Hooper, J.; Alnemrat, S.; Gaskell, K. J.; Eichhorn, B. Elucidation of the Fe(III) Gallate Structure in Historical Iron Gall Ink. Anal. Chem. 2016, 88, 5152-5158.

(4) Henniges, U.; Reibke, R.; Banik, G.; Huhsmann, E.; Hähner, U.; Prohaska, T.; Potthast, A. Iron Gall Ink-Induced Corrosion of Cellulose: Aging, Degradation and Stabilization. Part 2: Application on Historic Sample Material. Cellulose 2008, 15, 861-870.

(5) Potthast, A.; Henniges, U.; Banik, G. Iron Gall Ink-Induced Corrosion of Cellulose: Aging, Degradation and Stabilization. Part 1: Model Paper Studies. Cellulose 2008, 15, 849-859.

(6) Poggi, G.; Giorgi, R.; Toccafondi, N.; Katzur, V.; Baglioni, P. Hydroxide Nanoparticles for Deacidification and Concomitant Inhibition of Iron-Gall Ink Corrosion of Paper. Langmuir 2010, 26, 19084-19090.

(7) Malešič, J.; Šala, M.; Šelih, V. S.; Kočar, D. Evaluation of a Method for Treatment of Iron Gall Ink Corrosion on Paper. Cellulose 2014, 21, 2925-2936. 
(8) Li, J.; Gardner, H. How Domains Constrain Creativity - The Case of Traditional Chinese and Western Painting. Am. Behav. Sci. 1993, 37, 94-101.

(9) Hu, G.; Kang, J.; Ng, L. W. T.; Zhu, X.; Howe, R. C. T.; Jones, C. G.; Hersam, M. C.; Hasan, T. Functional Inks and Printing of Two-Dimensional Materials. Chem. Soc. Rev. 2018, 47, $3265-3300$

(10) Ariga, K.; Watanabe, S.; Mori, T.; Takeya, J. Soft 2D Nanoarchitectonics. NPG Asia Mater. 2018, 10, 90-106.

(11) Igarashi, C.; Murata, A.; Itoh, Y.; Subekti, D. R. G.; Takahashi, S.; Kamagata, K. DNA Garden: A Simple Method for Producing Arrays of Stretchable DNA for Single-Molecule Fluorescence Imaging of DNA-Binding Proteins. Bull. Chem. Soc. Jpn. 2017, 90, 34-43.

(12) Pandika, M. The Shifting Picture of Rewritable Paper. ACS Cent. Sci. 2019, 5, 571-574.

(13) Hermy, M.; Honnay, O.; Firbank, L.; Grashof-Bokdam, C.; Lawesson, J. E. An Ecological Comparison Between Ancient and Other Forest Plant Species of Europe, and the Implications for Forest Conservation. Biological Conservation 1999, 91, 9-22.

(14) Sheng, L.; Li, M.; Zhu, S.; Li, H.; Xi, G.; Li, Y. G.; Wang, Y.; Li, Q.; Liang, S.; Zhong, K.; Zhang, S. X. A. Hydrochromic Molecular Switches for Water-Jet Rewritable Paper. Nat. Commun. 2014, 5, 3044.

(15) Ma, Y.; She, P.; Zhang, K. Y.; Yang, H.; Qin, Y.; Xu, Z.; Liu, S.; Zhao, Q.; Huang, W. Dynamic Metal-Ligand Coordination for Multicolour and Water-Jet Rewritable Paper. Nat. Commun. 2018, 9, 3.

(16) Ge, J.; Goebl, J.; He, L.; Lu, Z.; Yin, Y. Rewritable Photonic Paper with Hygroscopic Salt Solution as Ink. Adv. Mater. 2009, 21, 4259-4264.

(17) Xi, G.; Sheng, L.; Du, J.; Zhang, J.; Li, M.; Wang, H.; Ma, Y.; Zhang, S. X. A. Water Assisted Biomimetic Synergistic Process and its Application in Water-Jet Rewritable Paper. Nat. Commun. 2018, 9, 4819. 
(18) Wang, W.; Feng, J.; Ye, Y.; Lyu, F.; Liu, Y. S.; Guo, J.; Yin, Y. Photocatalytic Color Switching of Transition Metal Hexacyanometalate Nanoparticles for High-Performance LightPrintable Rewritable Paper. Nano Lett. 2017, 17, 755-761.

(19) Yuan, W.; Sun, L.; Tang, H.; Wen, Y.; Jiang, G.; Huang, W.; Jiang, L.; Song, Y.; Tian, H.; Zhu, D. A Novel Thermally Stable Spironaphthoxazine and its Application in Rewritable High Density Optical Data Storage. Adv. Mater. 2005, 17, 156-160.

(20) Chen, L.; Weng, M.; Huang, F.; Zhang, W. Long-Lasting and Easy-to-Use Rewritable Paper Fabricated by Printing Technology. ACS Appl. Mater. Interfaces 2018, 10, 40149-40155. (21) Ejima, H.; Richardson, J. J.; Caruso, F. Metal-Phenolic Networks as a Versatile Platform to Engineer Nanomaterials and Biointerfaces. Nano Today 2017, 12, 136-148.

(22) Podsiadlo, P.; Liu, Z.; Paterson, D.; Messersmith, P. B.; Kotov, N. A. Fusion of Seashell Nacre and Marine Bioadhesive Analogs: High-Strength Nanocompoisite by Layer-by-Layer Assembly of Clay and L-3,4-Dihydroxyphenylaianine Polymer. Adv. Mater. 2007, 19, 949-955. (23) Zeng, H.; Hwang, D. S.; Israelachvili, J. N.; Waite, J. H. Strong Reversible Fe ${ }^{3+}$-Mediated Bridging Between Dopa-Containing Protein Films in Water. P. Natl. Acad. Sci. USA 2010, 107, $12850-12853$.

(24) Holten-Andersen, N.; Harrington, M. J.; Birkedal, H.; Lee, B. P.; Messersmith, P. B.; Lee, K. Y. C.; Waite, J. H. pH-Induced Metal-Ligand Cross-Links Inspired by Mussel Yield SelfHealing Polymer Networks with Near-Covalent Elastic Moduli. P. Natl. Acad. Sci. USA 2011, $108,2651-2655$.

(25) Yun, G.; Pan, S.; Wang, T. Y.; Guo, J.; Richardson, J. J.; Caruso, F. Synthesis of Metal Nanoparticles in Metal-Phenolic Networks: Catalytic and Antimicrobial Applications of Coated Textiles. Adv. Healthcare Mater. 2018, 7, 1700934.

(26) Park, J. P.; Song, I. T.; Lee, J.; Ryu, J. H.; Lee, Y.; Lee, H. Vanadyl-Catecholamine Hydrogels Inspired by Ascidians and Mussels. Chem. Mater. 2015, 27, 105-111. 
(27) Krogsgaard, M.; Andersen, A.; Birkedal, H. Gels and Threads: Mussel-Inspired One-Pot Route to Advanced Responsive Materials. Chem. Commun. 2014, 50, 13278-13281.

(28) Harrington, M. J.; Masic, A.; Holten-Andersen, N.; Waite, J. H.; Fratzl, P. Iron-Clad Fibers: A Metal-Based Biological Strategy for Hard Flexible Coatings. Science 2010, 328, 216220.

(29) Park, J. H.; Choi, S.; Moon, H. C.; Seo, H.; Kim, J. Y.; Hong, S. P.; Lee, B. S.; Kang, E.; Lee, J.; Ryu, D. H.; Choi, I. S. Antimicrobial Spray Nanocoating of Supramolecular Fe(III)Tannic Acid Metal-Organic Coordination Complex: Applications to Shoe Insoles and Fruits. Sci. Rep. 2017, 7, 6980.

(30) Park, T.; Kim, J. Y.; Cho, H.; Moon, H. C.; Kim, B. J.; Park, J. H.; Hong, D.; Park, J.; Choi, I. S. Artificial Spores: Immunoprotective Nanocoating of Red Blood Cells with Supramolecular Ferric Ion-Tannic Acid Complex. Polymers 2017, 9, 140.

(31) Guo, J.; Ping, Y.; Ejima, H.; Alt, K.; Meissner, M.; Richardson, J. J.; Yan, Y.; Peter, K.; von Elverfeldt, D.; Hagemeyer, C. E.; Caruso, F. Engineering Multifunctional Capsules through the Assembly of Metal-Phenolic Networks. Angew. Chem. Int. Ed. 2014, 53, 5546-5551.

(32) Sileika, T. S.; Barrett, D. G.; Zhang, R.; Lau, K. H. A.; Messersmith, P. B. Colorless Multifunctional Coatings Inspired by Polyphenols Found in Tea, Chocolate, and Wine. Angew. Chem. Int. Ed. 2013, 52, 10766-10770.

(33) Barrett, D. G.; Sileika, T. S.; Messersmith, P. B. Molecular Diversity in Phenolic and Polyphenolic Precursors of Tannin-Inspired Nanocoatings. Chem. Commun. 2014, 50, $7265-$ 7268 .

(34) Lee, J. S.; Lee, J. S.; Lee, M. S.; An, S.; Yang, K.; Lee, K.; Yang, H. S.; Lee, H.; Cho, S. W. Plant Flavonoid-Mediated Multifunctional Surface Modification Chemistry: Catechin Coating for Enhanced Osteogenesis of Human Stem Cells. Chem. Mater. 2017, 29, 4375-4384. (35) Cui, J.; Wang, Y.; Hao, J.; Caruso, F. Mesoporous silica-templated assembly of luminescent polyester particles. Chem. Mater. 2009, 21, 4310-4315. 
(36) Zhong, Q. Z.; Pan, S. J.; Rahim, M. A.; Yun, G.; Li, J.; Ju, Y.; Lin, Z.; Han, Y.; Ma, Y.; Richardson, J. J.; Caruso, F. Spray Assembly of Metal-Phenolic Networks: Formation, Growth, and Applications. ACS Appl. Mater. Interfaces 2018, 10, 33721-33729.

(37) Xu, H.; Nishida, J.; Ma, W.; Wu, H.; Kobayashi, M.; Otsuka, H.; Takahara, A. Competition between Oxidation and Coordination in Cross-Linking of Polystyrene Copolymer Containing Catechol Groups. ACS Macro Lett. 2012, 1, 457-460.

(38) Mandal, M.; Oppelt, K.; List, M.; Teasdale, I.; Chakraborty, D.; Monkowius, U. Copper(II) Complexes with Imino Phenoxide Ligands: Synthesis, Characterization, and their Application as Catalysts for the Ring-Opening Polymerization of rac-Lactide. Monatsh. Chem. 2016, 147, $1883-1892$.

(39) Negri, C.; Signorile, M.; Porcaro, N. G.; Borfecchia, E.; Berlier, G.; Janssens, T. V. W.; Bordiga, S. Dynamic $\mathrm{Cu}^{\mathrm{II}} / \mathrm{Cu}^{\mathrm{I}}$ Speciation in $\mathrm{Cu}-\mathrm{CHA}$ Catalysts by In Situ Diffuse Reflectance UV-vis-NIR Spectroscopy. Appl. Catal., A 2019, 578, 1-9.

(40) Kwolek, P.; Oszajca, M.; Szaciłowski, L. Catecholate and 2,3-Acenediolate Complexes of $\mathrm{d}^{0}$ Ions as Prospective Materials for Molecular Electronics and Spintronics. Coord. Chem. Rev. 2012, 256, 1706-1731.

(41) Vila, A.; Ferrer, N.; García, J. F. Chemical Composition of Contemporary Black Printing Inks Based on Infrared Spectroscopy: Basic Information for the Characterization and Discrimination of Artistic Prints. Anal. Chim. Acta 2007, 591, 97-105.

(42) Zięba-Palus, J.; Trzcińska, B. M. Establishing of Chemical Composition of Printing Ink. J. Forensic Sci. 2011, 56, 819-821.

(43) Samson, D. A.; Gradwell, M. H. S.; McGill, W. J. Oil-Dispersed Waxes for Printing Inks. Part II: Effect of Chemical Composition and Production Procedure on Particle Characteristics and Performance in Prints. Jocca-Surf. Coat. Int. 1999, 82, 280-284. 
TABLE OF CONTENTS

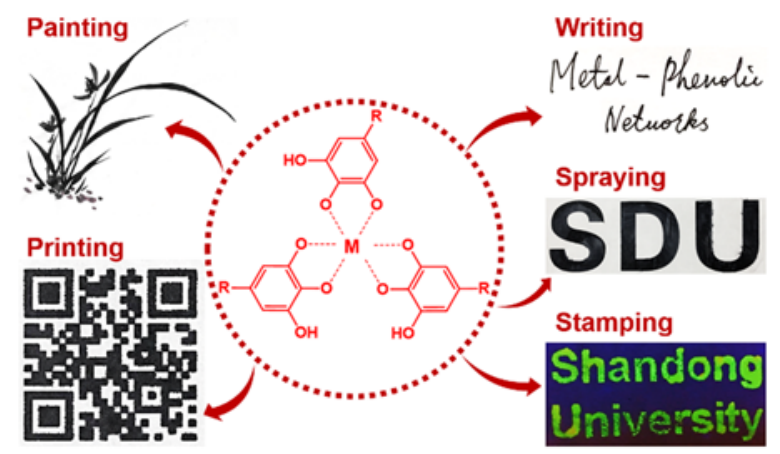

